\title{
Flat extensions of abstract polytopes
}

\author{
Gabe Cunningham \\ Department of Mathematics \\ University of Massachusetts Boston \\ Boston, Massachusetts, USA, 02125 \\ gabriel.cunningham@gmail.com
}

November 17, 2021

\begin{abstract}
We consider the problem of constructing an abstract $(n+1)$-polytope $\mathcal{Q}$ with $k$ facets isomorphic to a given $n$-polytope $\mathcal{P}$, where $k \geq 3$. In particular, we consider the case where we want $\mathcal{Q}$ to be $(n-2, n)$-flat, meaning that every $(n-2)$-face is incident to every $n$-face (facet). We show that if $\mathcal{P}$ admits such a flat extension for a given $k$, then the facet graph of $\mathcal{P}$ is $(k-1)$-colorable. Conversely, we show that if the facet graph is $(k-1)$-colorable and $k-1$ is prime, then $\mathcal{P}$ admits a flat extension for that $k$. We also show that if $\mathcal{P}$ is facet-bipartite, then for every even $k$, there is a flat extension $\mathcal{P} \mid k$ such that every automorphism of $\mathcal{P}$ extends to an automorphism of $\mathcal{P} \mid k$. Finally, if $\mathcal{P}$ is a facet-bipartite $n$-polytope and $\mathcal{Q}$ is a vertex-bipartite $m$-polytope, we describe a flat amalgamation of $\mathcal{P}$ and $\mathcal{Q}$, an $(m+n-1)$-polytope that is $(n-2, n)$-flat, with $n$-faces isomorphic to $\mathcal{P}$ and co- $(n-2)$-faces isomorphic to $\mathcal{Q}$.
\end{abstract}

Key Words: polytope, extension, amalgamation, perfect 1-factorization

AMS Subject Classification (2000): Primary: 52B05. Secondary: 52B11, 52B15.

\section{Introduction}

Fix an abstract $n$-polytope $\mathcal{P}$ and a positive integer $k$, and suppose that you want to glue together copies of $\mathcal{P}$ to build an $(n+1)$-polytope $\mathcal{Q}$ such that each $(n-2)$-face of $\mathcal{Q}$ is surrounded by $k$ copies of $\mathcal{P}$. What is the smallest possible $\mathcal{Q}$ ?

Clearly, the best we could hope for is to use only $k$ copies of $\mathcal{P}$, building $\mathcal{Q}$ so that every $(n-2)$-face is surrounded by every copy of $\mathcal{P}$. For which polytopes $\mathcal{P}$ and which integers $k$ is this possible? When $k=2$, this is always possible; this is called the trivial extension of $\mathcal{P}$. More generally, we will show that if $k$ is even, then this is always possible if $\mathcal{P}$ is facetbipartite (in other words, if we can color the facets with two colors such that adjacent facets 
have different colors). On the other hand, we will show that if $\mathcal{P}$ is not $(k-1)$-facet-colorable, then it is impossible to glue together $k$ copies of $\mathcal{P}$ in this manner.

The polytopes that we are working with are abstract polytopes, which are usually defined in terms of a poset that is similar to the face-lattice of a polytope [7]. For the constructions discussed here, it is more natural to consider polytopes as a subclass of maniplexes, which can be viewed as a kind of edge-colored graphs [10]. The paper [5] provides a characterization of which maniplexes are the flag graphs of polytopes, which is a key ingredient to our approach.

We start by giving some background on maniplexes and polytopes in Section 2. Then we consider the problem of building a flat extension of $\mathcal{P}$ that uses $k$ copies in Section 3 . Corollary 3.2 shows that the facet graph of $\mathcal{P}$ must be $(k-1)$-colorable. In Section 3.1, we will show that if $\mathcal{P}$ is facet-bipartite, then any even $k \geq 2$ will work (see Theorem 3.4) and we determine some further properties related to its automorphism group (see Proposition 3.9). Then, in Section 3.2, we describe a more general construction that works for any $\mathcal{P}$ whose facet graph is $(k-1)$-colorable, subject to some restrictions on $k$ (see Theorem 3.14). In Section 4, we generalize the first construction in another way, building a flat amalgamation of a facet-bipartite polytope $\mathcal{P}$ and a vertex-bipartite polytope $\mathcal{Q}$. Finally, we briefly discuss some open questions that remain in Section 5.

\section{$2 \quad$ Maniplexes and polytopes}

Abstract polytopes are posets that, broadly speaking, look something like the incidence relation of a convex polytope or a tiling of a surface or space. Their basic theory is outlined in [7]. Another way to view a polytope is in terms of its flag graph, and in [5], GarzaVargas and Hubard characterize which properly-edge-colored regular simple graphs are the flag graphs of abstract polytopes. Since the constructions in this paper operate on the flag graphs of polytopes, it will be natural for us to define polytopes in terms of graphs instead of posets.

Let us start with a (non-standard) definition. Let $\mathcal{G}$ be a graph whose nodes we will call flags. Then $\mathcal{G}$ is an $n$-pre-maniplex if it is an $n$-regular simple graph where the edges are colored $\{0,1, \ldots, n-1\}$ and each flag is incident to exactly one edge of each color. For each color $i$ and each flag $\Phi$, we define $\Phi^{i}$ to be the other endpoint of the edge of color $i$ that touches $\Phi$, and we say that $\Phi^{i}$ is $i$-adjacent to $\Phi$. We further define $\Phi^{i, j}$ to be $\left(\Phi^{i}\right)^{j}$.

If $\mathcal{G}$ is an $n$-pre-maniplex, then let $\mathcal{G}\left[i_{1}, \ldots, i_{m}\right]$ denote the subgraph of $\mathcal{G}$ with all of the same flags as $\mathcal{G}$ and with only the edges of colors $i_{1}, \ldots, i_{m}$. The $\left(i_{1}, \ldots, i_{m}\right)$-color-components of $\mathcal{G}$ are the connected components of $\mathcal{G}\left[i_{1}, \ldots, i_{m}\right]$.

In an $n$-pre-maniplex $\mathcal{G}$, we say that colors $i$ and $j$ commute if, for each flag $\Phi, \Phi^{i, j}=\Phi^{j, i}$. Equivalently, $i$ and $j$ commute if $\mathcal{G}[i, j]$ is a union of 4 -cycles. Note that if $A$ and $B$ are sets of colors such that every color in $A$ commutes with every color in $B$, then whenever there is a path from $\Phi$ to $\Psi$ using edges of colors in $A \cup B$, there must be a flag $\Lambda$ such that there is a path from $\Phi$ to $\Lambda$ using color set $A$ and then a path from $\Lambda$ to $\Psi$ using color set $B$.

We define an $n$-maniplex to be an $n$-pre-maniplex such that, for every pair of colors $i$ and $j$ such that $|i-j|>1$, those colors commute. For each $i \in\{0, \ldots, n-1\}$, the $i$-faces of 
an $n$-maniplex are the connected components of $\mathcal{G}[0, \ldots, i-1, i+1, \ldots, n-1]$. We say that two faces are incident if they have nonempty intersection. The $(n-1)$-faces of an $n$-maniplex are called its facets.

Finally, an $n$-maniplex is an n-polytope if it satisfies the following Path Intersection Property: for every pair of flags $\Phi$ and $\Psi$ and every $i<j$, if there is a path between $\Phi$ and $\Psi$ that uses colors $i, \ldots, n-1$ and another path between them that uses colors $0, \ldots, j$, then there must be a path between them that uses only the colors $i, \ldots, j$ (see [5, Thm. 5.3]).

In the context of graphs, an automorphism of an $n$-polytope is a graph automorphism that preserves the edge colors, and we denote the automorphism group of $\mathcal{P}$ by $\Gamma(\mathcal{P})$. In other words, $\varphi$ is an automorphism of $\mathcal{P}$ if it is a bijection on the flags such that, for every flag $\Phi$ and every edge color $i$, we have $\Phi^{i} \varphi=(\Phi \varphi)^{i}$. If $\mathcal{P}$ and $\mathcal{Q}$ are $n$-polytopes, then $\mathcal{P}$ covers $\mathcal{Q}$ if there is a surjective graph homomorphism from $\mathcal{P}$ to $\mathcal{Q}$ that preserves the edge colors. A polytope is regular if the automorphism group acts transitively on the flags. The symmetry type graph of a polytope $\mathcal{P}$ is the quotient of $\mathcal{P}$ by the orbits of the nodes under $\Gamma(\mathcal{P})$; see [3].

The facet graph of a polytope $\mathcal{P}$ is a simple graph whose nodes correspond to the facets of $\mathcal{P}$, and where two nodes are connected if the corresponding facets are connected by an edge labeled $n-1$ in $\mathcal{P}$. A polytope is facet-bipartite if its facet graph is bipartite. Equivalently, a polytope is facet-bipartite if and only if there are no cycles in $\mathcal{P}$ with an odd number of edges labeled $n-1$.

The dual of a polytope $\mathcal{P}$ is the polytope $\mathcal{P}^{*}$ obtained by changing every edge label from $i$ to $n-1-i$. The 1 -skeleton of $\mathcal{P}$ is the facet graph of $\mathcal{P}^{*}$. That is, the nodes of the 1 -skeleton correspond to the 0 -faces of $\mathcal{P}$, and two nodes are connected if there is an edge labeled 1 between the corresponding faces in $\mathcal{P}$. The polytope $\mathcal{P}$ is vertex-bipartite if there are no cycles in $\mathcal{P}$ with an odd number of edges labeled 0 .

A polytope $\mathcal{P}$ is $(i, j)$-flat if every $i$-face is incident to every $j$-face. In other words, $\mathcal{P}$ is $(i, j)$-flat if, for every flag $\Phi$ and every $j$-face, there is a path from $\Phi$ to some flag in that $j$-face that does not use any edges of color $i$.

Proposition 2.1. Suppose $i<j$. Then the n-polytope $\mathcal{P}$ is $(i, j)$-flat if and only if, for every flag $\Phi$ and every $j$-face, there is a path from $\Phi$ to some flag in that $j$-face that only uses edges of colors $\{i+1, \ldots, n-1\}$.

Proof. Suppose that $\mathcal{P}$ is $(i, j)$-flat and consider an arbitrary flag $\Phi$ and a $j$-face. Suppose that $\Psi$ is a flag in the $j$-face such that there is a path from $\Phi$ to $\Psi$ that never uses color $i$. So the path from $\Phi$ to $\Psi$ uses colors $\{0, \ldots, i-1\}$ and $\{i+1, \ldots, n-1\}$. Since these two color sets commute, there must be a flag $\Lambda$ such that there is a path from $\Phi$ to $\Lambda$ using colors $\{i+1, \ldots, n-1\}$ and then a path from $\Lambda$ to $\Psi$ using colors $\{0, \ldots, i-1\}$. Since $i<j$, the latter color set does not include $j$, and so $\Lambda$ is in the same $j$-face as $\Psi$. Then there is a path from $\Phi$ to the $j$-face that only uses edges of colors $\{i+1, \ldots, n-1\}$. That proves one direction, and the other direction is clear. 


\section{Flat extensions}

Our goal is to take $k$ copies of an $n$-polytope $\mathcal{P}$ and glue them together into an $(n+1)$ polytope $\mathcal{Q}$. Furthermore, we would like for every $(n-2)$-face of $\mathcal{Q}$ to be surrounded by all $k$ copies of $\mathcal{P}$ - in other words, we would like $\mathcal{Q}$ to be $(n-2, n)$-flat. How do we get started?

If such a polytope $\mathcal{Q}$ exists, then removing all edges labeled $n$ yields $k$ copies of $\mathcal{P}$. So in order to build $\mathcal{Q}$, let us take $k$ copies of $\mathcal{P}$ (which we will call the layers of $\mathcal{Q}$ ), labeled $\mathcal{P}_{1}, \ldots, \mathcal{P}_{k}$. For each flag $\Phi$ of $\mathcal{P}$, we will write $\Phi_{i}$ for the image of $\Phi$ in $\mathcal{P}_{i}$. Now, we create $\mathcal{Q}$ from these $k$ copies of $\mathcal{P}$ by adding a perfect matching using new edges labeled $n$. How do we do so in a way that ensures that $\mathcal{Q}$ is a polytope?

First we need to make sure that color $n$ commutes with each color $c$ in $\{0, \ldots, n-2\}$. To do so, once we decide to match some flag $\Phi_{i}$ to $\Psi_{j}$, we must also match $\left(\Phi_{i}\right)^{c}$ to $\left(\Psi_{j}\right)^{c}$ for every $c \in\{0, \ldots, n-2\}$. Applying this restriction recursively shows that the matching of flags must induce a matching of the $\{0, \ldots, n-2\}$-color components, which correspond to the facets of $\mathcal{P}$. (See Figure 1.)

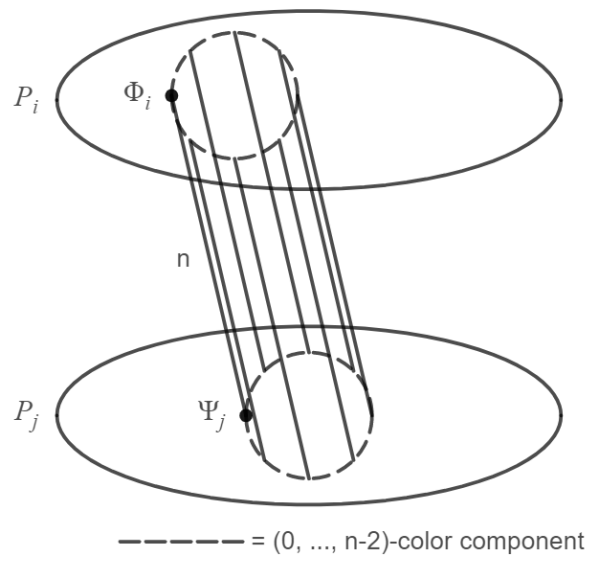

Figure 1: Matching $\Phi_{i}$ to $\Psi_{j}$ induces a matching of the $(0, \ldots, n-2)$-color components.

Next, we want $\mathcal{Q}$ to be $(n-2, n)$-flat. By Proposition 2.1 , this is equivalent to making every $(n-1, n)$-color-component intersect every $\mathcal{P}_{j}$.

We have already observed that once we match a flag $\Phi$, that induces a matching of $\Phi^{c}$ for each $c \in\{0, \ldots, n-2\}$. Now we will see that requiring that $\mathcal{Q}$ be flat restricts our choice of how we match $\Phi^{n-1}$.

Proposition 3.1. Suppose $\mathcal{Q}$ is an $(n+1)$-polytope that is $(n-2, n)$-flat, with $k$ facets isomorphic to $\mathcal{P}$, where $k \geq 3$. Then for every $\Phi_{i}$, the flags $\left(\Phi_{i}\right)^{n}$ and $\left(\Phi_{i}\right)^{n-1, n}$ are in different layers $\mathcal{P}_{j}$.

Proof. Suppose $\left(\Phi_{i}\right)^{n}$ and $\left(\Phi_{i}\right)^{n-1, n}$ are in the same layer. Then there is a path from $\left(\Phi_{i}\right)^{n}$ to $\left(\Phi_{i}\right)^{n-1, n}$ using edges labeled $\{0, \ldots, n-1\}$. There is also a path from $\left(\Phi_{i}\right)^{n}$ to $\left(\Phi_{i}\right)^{n-1, n}$ 
using edges labeled only $n-1$ and $n$. Then the Path Intersection Property implies that there is a path using only edges labeled $n-1$, which means that $\left(\Phi_{i}\right)^{n, n-1}=\left(\Phi_{i}\right)^{n-1, n}$. Thus the $(n-1, n)$-color component that contains $\Phi_{i}$ consists of only four flags in two layers, and since $k \geq 3$ this implies that $\mathcal{Q}$ is not $(n-2, n)$-flat.

Let us reinterpret this result in terms of the facet graph of $\mathcal{P}$. For each facet of $\mathcal{P}$ (corresponding to a $(0, \ldots, n-2)$-color component of $\mathcal{Q})$, consider the flags in the last layer $\mathcal{P}_{k}$ that contain that facet. By the discussion earlier, all of these flags are matched to flags in some single layer $\mathcal{P}_{i}$ with $i \in\{1, \ldots, k-1\}$. Then we may color each facet of $\mathcal{P}$ by that number $i$, and Proposition 3.1 implies that this is a proper coloring! Therefore,

Corollary 3.2. Let $k \geq 3$. If $\mathcal{P}$ is an n-polytope such that its facet graph is not $(k-1)$ colorable, then there are no $(n+1)$-polytopes $\mathcal{Q}$ with $k$ facets isomorphic to $\mathcal{P}$ such that $\mathcal{Q}$ is $(n-2, n)$-flat.

Example 3.3. Since the facet graph of the $n$-simplex is the complete graph $K_{n+1}$, there are no $(n-2, n)$-flat $(n+1)$-polytopes $\mathcal{Q}$ with $n+1$ simplicial facets.

\subsection{Flat extensions of facet-bipartite polytopes}

When trying to define a matching in order to build $\mathcal{Q}$, the most straightforward way would be for each $\Phi_{i}$ to be matched to some $\Phi_{j}$. That is, each flag is matched to the 'same' flag in a different layer. The easiest such matching would have each flag $\Phi_{i}$ matched to either $\Phi_{i-1}$ or $\Phi_{i+1}$. Then the argument for Corollary 3.2 works in essentially the same way to show that, since each layer is matched to only two other layers, $\mathcal{P}$ must be facet-bipartite in order for this to work. We will show that this necessary condition is also sufficient.

So, suppose that $\mathcal{P}$ is a facet-bipartite $n$-polytope, and let $k$ be an even positive integer. Given a proper coloring of the facet graph of $\mathcal{P}$ with two colors (say red and blue), we can color each flag of $\mathcal{P}$ according to the color of its facet. Then, for each red flag $\Phi$, we will match $\Phi_{1}$ to $\Phi_{2}, \Phi_{3}$ to $\Phi_{4}$, and so on. For each blue flag $\Psi$, we will match $\Psi_{2}$ to $\Psi_{3}, \Psi_{4}$ to $\Psi_{5}$, and so on (matching $\Psi_{k}$ to $\Psi_{1}$ ). We refer to the graph that we obtain by $\mathcal{P} \mid k$. (See Figure 2.)

First, let us show that this construction really yields a polytope with the desired properties.

Theorem 3.4. The graph $\mathcal{P} \mid k$ is the flag graph of an $(n-2, n)$-flat $(n+1)$-polytope with $k$ facets isomorphic to $\mathcal{P}$.

Proof. By construction, it is clear that $\mathcal{P} \mid k$ has $k$ facets isomorphic to $\mathcal{P}$. If $\Phi$ is a red flag and $\Psi=\Phi^{n-1}$, then $\Psi$ is blue and the $(n-1, n)$-color component that contains $\Phi_{1}$ is the cycle

$$
\left(\Phi_{1}, \Phi_{2}, \Psi_{2}, \Psi_{3}, \Phi_{3}, \Phi_{4}, \ldots, \Psi_{k}, \Psi_{1}\right),
$$

which intersects every layer. It is clear then that each $(n-1, n)$-color component intersects every $\mathcal{P}_{i}$, and so $\mathcal{P} \mid k$ is $(n-2, n)$-flat. It is also clear that $\mathcal{P} \mid k$ is a maniplex, since we forced the new edges labeled $n$ to commute with the edges labeled $0,1, \ldots, n-2$. 


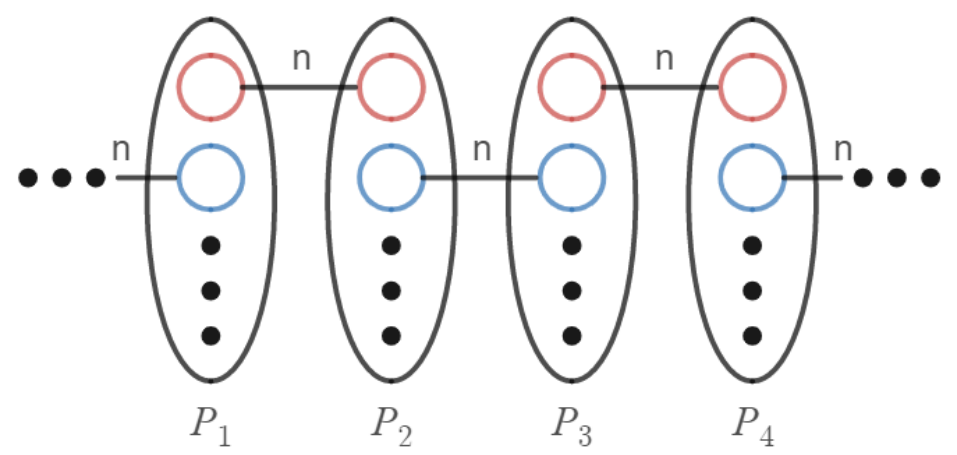

Figure 2: Flags are matched according to the coloring of the facet graph of $\mathcal{P}$.

It remains to show that $\mathcal{P} \mid k$ is a polytope by showing that it satisfies the Path Intersection Property. Consider colors $i$ and $j$ satisfying $0 \leq i<j \leq n$. Suppose there are two flags such that there is a path between them using colors $0, \ldots, j-1$ and $i+1, \ldots, n$. Since $j-1<n$, it follows that the two flags are in the same layer, and without loss of generality we will assume they are in layer 1 . So there are two flags $\Phi_{1}$ and $\Psi_{1}$ such that there is a path between them that uses colors $i+1, \ldots, n$. Since edges of color $n$ always connect two flags with the same underlying flag in $\mathcal{P}$, such a path induces a path between $\Phi$ and $\Psi$ in $\mathcal{P}$ that only uses colors $i+1, \ldots, n-1$. Similarly, there is an induced path between $\Phi$ and $\Psi$ in $\mathcal{P}$ that uses colors $0, \ldots, j-1$. Then, since $\mathcal{P}$ is a polytope, it follows that there is a path from $\Phi$ to $\Psi$ that uses colors $i+1, \ldots, j-1$, and then this path also lifts to an isomorphic path from $\Phi_{1}$ to $\Psi_{1}$ using only those colors, as desired.

Example 3.5. If $\mathcal{P}$ is the unique 1-polytope, then $\mathcal{P} \mid k$ is a $k$-gon.

Example 3.6. If $\mathcal{P}$ is a square, then $\mathcal{P} \mid 4$ is the map $\{4,4\}_{(2,0)}$ on the torus (see [7, Sec. $1 D])$.

Example 3.7. If $k=2$, then we don't even need for $\mathcal{P}$ to be facet-bipartite - we can just match each $\Phi_{1}$ to $\Phi_{2}$. Indeed, $\mathcal{P} \mid 2$ is the trivial extension of $\mathcal{P}$, also denoted $\{\mathcal{P}, 2\}$.

Example 3.8. Nothing goes wrong if we try $k=\infty$ and index the layers $\mathcal{P}_{i}$ by letting $i$ be any integer. We still get an $(n-2, n)$-flat polytope with infinitely many facets isomorphic to $\mathcal{P}$.

Now let us determine the automorphism group of $\mathcal{P} \mid k$. Fix a base flag $\Phi$ of $\mathcal{P}$, and consider an automorphism $\varphi$ of $\mathcal{P}$ that sends $\Phi$ to $\Psi$. Can we extend $\varphi$ to an automorphism $\tilde{\varphi}$ of $\mathcal{P} \mid k ?$

Without loss of generality, let us assume that $\Phi$ is red. Then the other red flags are those that can be reached from $\Phi$ using an even number of edges labeled $n-1$, and the blue flags are those that can be reached from $\Phi$ using an odd number of edges labeled $n-1$. Furthermore, $\varphi$ respects these color classes since, for each flag $\Lambda$, we have $\Lambda^{n-1} \varphi=(\Lambda \varphi)^{n-1}$. 
Now, if $\Psi$ is also red, then $\varphi$ preserves the color of every flag. Then we define $\tilde{\varphi}$ so that, for each flag $\Lambda$ of $\mathcal{P}$,

$$
\left(\Lambda_{i}\right) \tilde{\varphi}=(\Lambda \varphi)_{i}
$$

In other words, $\tilde{\varphi}$ fixes each layer setwise, and acts on each layer in the same way that $\varphi$ acts on $\mathcal{P}$. To see that this defines an automorphism, it suffices to show that $\tilde{\varphi}$ preserves the edges of color $n$, and this is true since

$$
\left(\Lambda_{i}\right)^{n} \tilde{\varphi}=\Lambda_{i \pm 1} \tilde{\varphi}=(\Lambda \varphi)_{i \pm 1}=\left((\Lambda \varphi)_{i}\right)^{n}=\left(\Lambda_{i} \tilde{\varphi}\right)^{n} .
$$

If $\Psi$ is blue instead, then the action of $\varphi$ on $\mathcal{P}$ reverses the color of every flag. Then we define $\tilde{\varphi}$ so that, for each flag $\Lambda$ of $\mathcal{P}$,

$$
\left(\Lambda_{i}\right) \tilde{\varphi}=(\Lambda \varphi)_{k+2-i}
$$

Again, this will define an automorphism if and only if $\tilde{\varphi}$ preserves the edges of color $n$, and this is true since

$$
\left(\Lambda_{i}\right)^{n} \tilde{\varphi}=\Lambda_{i \pm 1} \tilde{\varphi}=(\Lambda \varphi)_{k+2-i \mp 1}=\left((\Lambda \varphi)_{k+2-i}\right)^{n}=\left(\Lambda_{i} \tilde{\varphi}\right)^{n},
$$

where the third equality follows because $\Lambda \varphi$ is the opposite color of $\Lambda$, and so the matching of $\Lambda \varphi$ is in the opposite direction of the matching of $\Lambda$ (that is, $\mp$ instead of \pm ). So in either case, we see that each automorphism of $\mathcal{P}$ lifts to an automorphism of $\mathcal{P} \mid k$; in other words, $\mathcal{P} \mid k$ is hereditary (see [8]).

In addition to these automorphisms $\tilde{\varphi}$, which all fix the first layer setwise, there are automorphisms of $\mathcal{P} \mid k$ that simply permute the layers. Indeed, it is clear from the symmetry of the graph (see Figure 2) that there is an automorphism $\alpha$ that sends each $\Lambda_{i}$ to $\Lambda_{k+3-i}$ and an automorphism $\beta$ that sends each $\Lambda_{i}$ to $\Lambda_{k+5-i}$ (with the subscripts of $\Lambda$ reduced modulo $k$ ). The subgroup $\langle\alpha, \beta\rangle$ acts transitively on the layers, and the orbit of the flag $\Lambda_{1}$ is all flags $\Lambda_{j}$.

We can now characterize the automorphism group of $\mathcal{P} \mid k$.

Proposition 3.9. Let $\mathcal{P}$ be a facet-bipartite $n$-polytope and let $k$ be a positive even integer. Let $\tilde{\varphi}, \alpha$ and $\beta$ be defined as above.

(a) $\mathcal{P} \mid k$ is hereditary.

(b) $\Gamma(\mathcal{P} \mid k) \cong \Gamma(\mathcal{P}) \ltimes\langle\alpha, \beta\rangle$.

(c) The symmetry type graph of $\mathcal{P} \mid k$ is obtained from the symmetry type graph of $\mathcal{P}$ by adding semi-edges labeled $n$ to each node. In particular, $\mathcal{P} \mid k$ is regular if and only if $\mathcal{P}$ is regular.

Proof. The first part was already proved in the previous discussion. For the second part, let us first show that every automorphism in $\Gamma(\mathcal{P} \mid k)$ may be written as $\tilde{\varphi} \gamma$, with $\varphi \in \Gamma(\mathcal{P})$ and $\gamma \in\langle\alpha, \beta\rangle$. Fix a base flag $\Phi$ of $\mathcal{P}$, and suppose that an automorphism $\psi$ of $\mathcal{P} \mid k$ sends $\Phi_{1}$ 
to $\Psi_{j}$. Then there must be an automorphism $\varphi$ of $\mathcal{P}$ that sends $\Phi$ to $\Psi$, and the induced automorphism $\tilde{\varphi}$ sends $\Phi_{1}$ to $\Psi_{1}$. Then there is some $\gamma \in\langle\alpha, \beta\rangle$ that sends $\Psi_{1}$ to $\Psi_{j}$, and so $\tilde{\varphi} \gamma$ sends $\Phi_{1}$ to $\Psi_{j}$. Since polytope automorphisms are determined by their action on any one flag, this shows that $\psi=\tilde{\varphi} \gamma$.

Next, we note that $\alpha$ and $\beta$ both only change the subscript of a flag independently of the underlying flag of $\mathcal{P}$. Similarly, $\tilde{\varphi}$ only changes the underlying flag, independent of the subscript. So if $\gamma \in\langle\alpha, \beta\rangle$, then $\tilde{\varphi}^{-1} \gamma \tilde{\varphi}$ also only changes the subscript of each flag independently of the underlying flag, and so $\tilde{\varphi}^{-1} \gamma \tilde{\varphi} \in\langle\alpha, \beta\rangle$. So $\langle\alpha, \beta\rangle$ is normal in $\Gamma(\mathcal{P} \mid k)$. Finally, since each $\tilde{\varphi}$ fixes the first layer setwise, whereas no nontrivial element of $\langle\alpha, \beta\rangle$ fixes the first layer, we find that $\langle\alpha, \beta\rangle \cap \Gamma(\mathcal{P})=\langle 1\rangle$, and so $\Gamma(\mathcal{P} \mid k) \cong \Gamma(\mathcal{P}) \ltimes\langle\alpha, \beta\rangle$.

For the last part, note that the orbit of each $\Lambda_{i}$ under $\langle\alpha, \beta\rangle$ consists of all $k$ flags of the form $\Lambda_{j}$, and so these flags are all identified under the quotient by $\Gamma(\mathcal{P} \mid k)$. In particular, each flag is in the same orbit as its $n$-adjacent flag. Furthermore, any pair of flags $\Phi_{i}$ and $\Psi_{j}$ that lie in the same orbit must have underlying flags $\Phi$ and $\Psi$ that lie in the same orbit of $\Gamma(\mathcal{P})$, and so the symmetry type graph of $\mathcal{P} \mid k$ is just the symmetry type graph of $\mathcal{P}$ with extra semi-edges labeled $n$ at each node.

Let us now show some nice properties of $\mathcal{P} \mid k$ related to covers.

Proposition 3.10. If $\mathcal{P}$ and $\mathcal{Q}$ are facet-bipartite polytopes such that $\mathcal{Q}$ covers $\mathcal{P}$, then $\mathcal{Q} \mid k$ covers $\mathcal{P} \mid k$ for every even positive integer $k$.

Proof. To say that $\mathcal{Q}$ covers $\mathcal{P}$ is to say that there is a color-preserving graph epimorphism $\varphi$ from $\mathcal{Q}$ to $\mathcal{P}$. Fix a flag $\Psi$ of $\mathcal{Q}$ and let $\Phi=(\Psi) \varphi$. Without loss of generality, we may color both $\Phi$ and $\Psi$ red, so that $\Phi_{1}$ is matched to $\Phi_{2}$ and $\Psi_{1}$ is matched to $\Psi_{2}$. Then the obvious extension of $\varphi$ that acts separately on each layer of $\mathcal{Q} \mid k$ will also respect the edges of color $n$, and thus $\mathcal{Q} \mid k$ covers $\mathcal{P} \mid k$.

Proposition 3.11. If $\mathcal{P}$ is a facet-bipartite polytope and $k_{1}$ and $k_{2}$ are positive even integers with $k_{2}$ a multiple of $k_{1}$, then $\mathcal{P} \mid k_{2}$ covers $\mathcal{P} \mid k_{1}$. In particular, for every even positive integer $k$, the polytope $\mathcal{P} \mid k$ covers the trivial extension $\mathcal{P} \mid 2$.

Proof. The function taking each $\Phi_{i}$ to $\Phi_{i\left(\bmod k_{1}\right)}$ is a color-preserving graph epimorphism.

Next, we note that it is possible to repeatedly apply this construction:

Proposition 3.12. If $\mathcal{P}$ is a facet-bipartite n-polytope, then for every finite sequence $k_{1}, \ldots, k_{m}$ with each $k_{i}$ a positive even integer, there is a facet-bipartite polytope $\mathcal{Q}=\mathcal{P}\left|k_{1}\right| k_{2}|\cdots| k_{m}$ that is $(i, i+2)$-flat for each $i$ in $\{n-2, \ldots, n+m-3\}$. Furthermore, $\mathcal{Q}$ is regular if $\mathcal{P}$ is regular.

Proof. The first part follows immediately from the fact that the facet graph of $\mathcal{P} \mid k$ is an even cycle (consisting of the $k$ layers $\mathcal{P}_{i}$ ), and so $\mathcal{P} \mid k$ is facet-bipartite. The second part follows from Proposition 3.9(c).

Example 3.13. For any sequence of positive even integers $k_{1}, \ldots, k_{m}$, we can take $\mathcal{P}$ to be a $k_{1}$-gon and then extend it by $k_{2}, \ldots, k_{m}$. This yields a regular $(m+1)$-polytope that is $(i, i+2)$-flat for each $i$ in $\{0, \ldots, m-1\}$. In fact, this is a tight polytope of type $\left\{k_{1}, \ldots, k_{m}\right\}$; see [2]. 


\subsection{Flat extensions of other polytopes}

We have seen that if $\mathcal{P}$ is facet-bipartite, then there is a straightforward matching on $k$ copies of $\mathcal{P}$ that yields a polytope $\mathcal{P} \mid k$. What can we do with other polytopes $\mathcal{P}$ ? Let us fix an even $k \geq 4$ and try to build an $(n-2, n)$-flat $(n+1)$-polytope $\mathcal{Q}$ with $k$-facets isomorphic to $\mathcal{P}$. As before, we will focus on the case where each flag $\Phi_{i}$ is matched to some $\Phi_{j}$.

First, recall that Corollary 3.2 says that in order for $\mathcal{Q}$ to exist, $\mathcal{P}$ must be $(k-1)$-facetcolorable. Naturally, we wonder whether this necessary condition is also sufficient. Suppose $\mu$ is a proper coloring of the facet graph of $\mathcal{P}$, with colors $1,2, \ldots, k-1$, (though some colors may not be used). As before, we can extend this to a (non-proper) coloring of $\mathcal{P}$ itself by coloring each flag according to the color of its facet. Take $k$ copies of $\mathcal{P}$ as before: $\mathcal{P}_{1}, \ldots, \mathcal{P}_{k}$, with each $\Phi_{i}$ colored the same as $\Phi$. For each color $c$, we designate a perfect matching $\sigma_{c}$ of the layers, and if $\Phi$ is color $c$, then we match $\Phi_{i}$ to $\Phi_{\sigma_{c}(i)}$. Since $\mu$ is a proper coloring of the facet graph, this ensures that flags in $\mathcal{P}_{k}$ that are $(n-1)$-adjacent are matched to distinct layers, as required (see Proposition 3.1).

To determine whether the matchings $\sigma_{c}$ satisfy the desired properties, it is helpful to represent them using a new graph called the layer graph. This is a graph on $k$ nodes, corresponding to the $k$ layers $\mathcal{P}_{1}, \ldots, \mathcal{P}_{k}$, where there is an edge of color $c$ between two nodes if $\sigma_{c}$ matches the corresponding layers. See Figure 3 for an example with $k=6$.

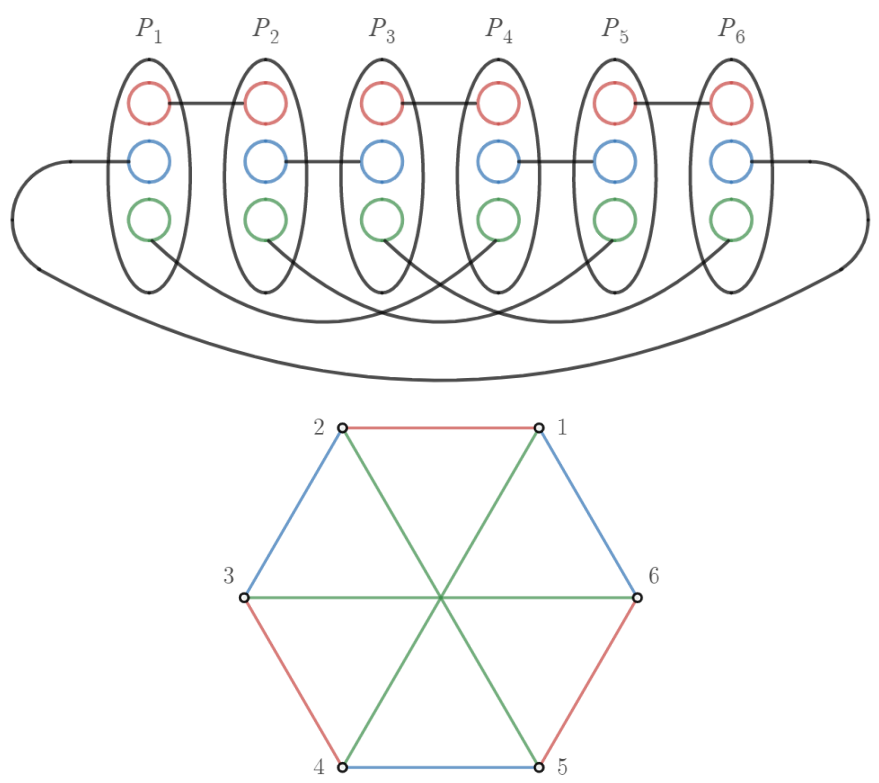

Figure 3: A matching of layers by color (above) and the corresponding layer graph (below).

Our goal is to pick matchings so that we obtain an $(n-2, n)$-flat $(n+1)$-polytope. Recall that to be $(n-2, n)$-flat means that, for every flag $\Phi_{i}$, the cycle that starts from $\Phi_{i}$ and follows edges labeled $n-1$ and $n$ should intersect every layer. Note that such a cycle consists only of flags of the form $\Phi_{j}$ and $\Psi_{j}$, where $\Psi=\Phi^{n-1}$. Therefore, the cycle is completely 
determined by the matchings corresponding to the colors of $\Phi$ and $\Psi$. Thus, if every pair of matchings of the layers yields a single cycle, then the result will be $(n-2, n)$-flat. In terms of the layer graph, this means that it suffices for every pair of colors to yield a single cycle. Such a collection of matchings is called a perfect 1-factorization of the graph. Kotzig conjectured in 1964 that every complete graph on an even number of vertices has a perfect 1-factorization [6]. This conjecture remains open; see [9] for a recent survey on this and related problems.

In any case, let us suppose that the complete graph $K_{k}$ admits a perfect 1 -factorization, and match flags $\Phi_{i}$ accordingly. As discussed, this will give us something that is $(n-2, n)$-flat. We still need to demonstrate that it is a polytope.

Theorem 3.14. Let $k$ be a positive even integer, $k \geq 4$, and let $\mathcal{P}$ be $(k-1)$-facet-colorable. Suppose that the complete graph $K_{k}$ has a perfect 1-factorization. Then the preceding construction defines the flag graph of a polytope.

Proof. Let $\mathcal{G}$ be the graph defined above. First, let us show that it is connected. The facet graph of $\mathcal{P}$ must use at least two colors, and by construction, the matchings corresponding to those two colors must induce a cycle that intersects each layer. Since each layer is connected, this shows that $\mathcal{G}$ is itself connected.

The remainder of the proof is analogous to the proof of Theorem 3.4. The key element is that each $\Phi_{i}$ is matched to some $\Phi_{j}$ - that is, each flag is matched to "itself" in another layer.

Example 3.15. If $k-1$ is prime or $k / 2$ is prime, then there is a perfect 1-factorization of $K_{k}$; see [6] and [1], respectively. Thus, every finite polytope $\mathcal{P}$ has infinitely many flat extensions - simply take $k-1$ to be a prime that is greater than or equal to the number of facets of $\mathcal{P}$.

\section{Flat amalgamations}

There is another way of thinking about $\mathcal{P} \mid k$ that readily admits one last generalization. It starts with seeing $\mathcal{P} \mid k$ as a mix of $\mathcal{P}$ with the flag graph of a $k$-gon. A similar construction for regular polytopes was described in $[7$, Sec. $4 \mathrm{~F}]$, using their automorphism groups instead of their flag graphs. For non-regular polytopes, the construction may provide different results depending on the choice of a base flag, and so we define the construction using rooted polytopes $(\mathcal{P}, \Phi)($ see $[4])$.

Definition 4.1. Suppose that $\mathcal{P}$ is an n-polytope with base flag $\Phi$ and that $\mathcal{Q}$ is an $m$ polytope with base flag $\Psi$. Let $0 \leq r \leq n-1$ with also $r \geq n-m$. Then the $r$-mix of $(\mathcal{P}, \Phi)$ with $(\mathcal{Q}, \Psi)$, denoted $(\mathcal{P}, \Phi) \diamond_{r}(\mathcal{Q}, \Psi)$, is the connected, properly edge-colored, $(m+r)$-regular graph $\mathcal{M}$ defined as follows.

(a) The base flag of $\mathcal{M}$ is the pair $(\Phi, \Psi)$. 
(b) For each $i \in\{0, \ldots, m+r-1\}$ and for each flag $(\Lambda, \Delta)$ of $\mathcal{M}$, we define $(\Lambda, \Delta)^{i}$ to be $\left(\Lambda^{i}, \Delta^{i-r}\right)$, with the understanding that if a superscript is "out of bounds" then we treat it as empty. In other words:

$$
(\Lambda, \Delta)^{i}=\left\{\begin{array}{ll}
\left(\Lambda^{i}, \Delta\right) & \text { if } 0 \leq i<r \\
\left(\Lambda^{i}, \Delta^{i-r}\right) & \text { if } r \leq i \leq n-1 \\
\left(\Lambda, \Delta^{i-r}\right) & \text { if } n \leq i \leq m+r-1
\end{array} .\right.
$$

(c) The flags of $\mathcal{M}$ are all pairs $(\Lambda, \Delta)$ (with $\Lambda$ a flag of $\mathcal{P}$ and $\Delta$ a flag of $\mathcal{Q}$ ) that are in the same connected component as $(\Phi, \Psi)$.

Definition 4.2. Suppose that $\mathcal{P}$ is an n-polytope and that $\mathcal{Q}$ is an m-polytope. Then the flat amalgamation of $(\mathcal{P}, \Phi)$ with $(\mathcal{Q}, \Psi)$ is $(\mathcal{P}, \Phi) \diamond_{n-1}(\mathcal{Q}, \Psi)$, denoted $(\mathcal{P}, \Phi) \mid(\mathcal{Q}, \Psi)$. If the base flags are understood in context, then we simply write $\mathcal{P} \mid \mathcal{Q}$. Note that, for each $i \in\{0, \ldots, m+n-2\}$ and for each flag $(\Lambda, \Delta)$,

$$
(\Lambda, \Delta)^{i}=\left\{\begin{array}{ll}
\left(\Lambda^{i}, \Delta\right) & \text { if } 0 \leq i<n-1, \\
\left(\Lambda^{n-1}, \Delta^{0}\right) & \text { if } i=n-1, \\
\left(\Lambda, \Delta^{i-n+1}\right) & \text { if } n \leq i \leq m+n-2
\end{array} .\right.
$$

Recall that $\mathcal{P}$ is facet-bipartite if and only if there are no cycles in $\mathcal{P}$ with an odd number of edges labeled $n-1$, and that $\mathcal{Q}$ is vertex-bipartite if and only if there are no cycles in $\mathcal{Q}$ with an odd number of edges labeled 0 .

Proposition 4.3. Let $\mathcal{P}$ be an n-polytope with base flag $\Phi$ and let $\mathcal{Q}$ be an m-polytope with base flag $\Psi$. Let $\mathcal{M}=\mathcal{P} \mid \mathcal{Q}$.

(a) Each connected component of $\mathcal{M}[0, \ldots, n-1]$ is isomorphic to $\mathcal{P}$ if and only if $\mathcal{P}$ is facet-bipartite.

(b) Each connected component of $\mathcal{M}[n-1, \ldots, m+n-2]$ is isomorphic to $\mathcal{Q}$ (with edge labels increased by $n-1)$ if and only if $\mathcal{Q}$ is vertex-bipartite.

Proof. Without loss of generality, consider the connected component of $\mathcal{M}[0, \ldots, n-1]$ that contains $(\Phi, \Psi)$. Recall that for $i<n-1$ we have that $(\Lambda, \Delta)^{i}=\left(\Lambda^{i}, \Delta\right)$, and so each flag in this connected component has either the form $(\Lambda, \Psi)$ or $\left(\Lambda, \Psi^{0}\right)$. Now let $\pi: \mathcal{M} \rightarrow \mathcal{P}$ be the projection in the first coordinate, sending each $(\Lambda, \Delta)$ to $\Lambda$. Since $\mathcal{P}$ is an $n$-polytope and we have edges of labels 0 through $n-1, \pi$ is surjective. Furthermore, $\pi$ will be injective (and thus bijective) if and only if there is no flag $\Lambda$ such that both $(\Lambda, \Psi)$ and $\left(\Lambda, \Psi^{0}\right)$ are in the connected component. A path from $(\Lambda, \Psi)$ to $\left(\Lambda, \Psi^{0}\right)$ exists if and only if there is a cycle in $\mathcal{P}$ that includes $\Lambda$ and has an odd number of edges labeled $n-1$. Thus, $\pi$ is bijective if and only if no such cycle exists, which is to say if and only if $\mathcal{P}$ is facet-bipartite.

The proof of the second part is analogous. 
In the usual language of polytopes, we say that if $\mathcal{P}$ is facet-bipartite and $\mathcal{Q}$ is vertexbipartite, then the $n$-faces of $\mathcal{P} \mid \mathcal{Q}$ are isomorphic to $\mathcal{P}$ and the co- $(n-2)$-faces are isomorphic to $\mathcal{Q}$.

We now collect a few properties of $\mathcal{P} \mid \mathcal{Q}$. Let $\mathcal{F}(\mathcal{M})$ denote the set of flags of the maniplex $\mathcal{M}$. As in Section 3.1, we can properly color the facet graph of $\mathcal{P}$ with two colors, and then extend this coloring to the flag graph. Similarly, we can properly color the 1 -skeleton of $\mathcal{Q}$ with two colors and extend this coloring to the flag graph.

Proposition 4.4. Let $\mathcal{P}$ be a facet-bipartite n-polytope with base flag $\Phi$ and let $\mathcal{Q}$ be a vertex-bipartite $m$-polytope with base flag $\Psi$. Color the flags of $\mathcal{P}$ red and blue according to a bipartition of its facet graph, and color the flags of $\mathcal{Q}$ red and blue according to a bipartition of its 1-skeleton, and let us assume that $\Phi$ and $\Psi$ are both red.

(a) $\mathcal{F}(\mathcal{P} \mid \mathcal{Q})=\{(\Lambda, \Delta) \in \mathcal{F}(\mathcal{P}) \times \mathcal{F}(\mathcal{Q}): \Lambda$ and $\Delta$ are the same color $\}$.

(b) $|\mathcal{F}(\mathcal{P} \mid \mathcal{Q})|=\frac{1}{2}|\mathcal{F}(\mathcal{P})| \cdot|\mathcal{F}(\mathcal{Q})|$.

(c) $\mathcal{P} \mid \mathcal{Q}$ is $(n-2, n)$-flat.

Proof. Suppose that $(\Lambda, \Delta)$ is a flag of $\mathcal{P} \mid \mathcal{Q}$. By the definition of $(\Lambda, \Delta)^{j}$, either both components change color (when $j=n-1$ ) or neither component changes color. Since $\mathcal{P} \mid \mathcal{Q}$ consists of only those flags that are reachable from $(\Phi, \Psi)$, which are both red, it follows that all flags of $\mathcal{P} \mid \mathcal{Q}$ have the same color in both components.

Now, suppose that $\Lambda$ and $\Delta$ are arbitrary flags of $\mathcal{P}$ and $\mathcal{Q}$ (respectively) that are the same color. There is a path in $\mathcal{P}$ from $\Phi$ to $\Lambda$, and this induces a path in $\mathcal{P} \mid \mathcal{Q}$ that uses only edges of colors in $\{0, \ldots, n-1\}$. Such a path will either take $(\Phi, \Psi)$ to $(\Lambda, \Psi)$ or to $\left(\Lambda, \Psi^{0}\right)$. In the latter case, we may follow an additional edge labeled $n-1$ to arrive at $\left(\Lambda^{n-1}, \Psi\right)$. Now, there is a path in $\mathcal{Q}$ from $\Psi$ to $\Delta$, and this induces a path in $\mathcal{P} \mid \mathcal{Q}$ that uses only edges of colors in $\{n-1, \ldots, m+n-2\}$. Such a path will take us from $(\Lambda, \Psi)$ or $\left(\Lambda^{n-1}, \Psi\right)$ to $(\Lambda, \Delta)$ or $\left(\Lambda^{n-1}, \Delta\right)$. By the previous paragraph, since $\Lambda^{n-1}$ has a different color to $\Delta$, the flag $\left(\Lambda^{n-1}, \Delta\right)$ cannot be in $\mathcal{P} \mid \mathcal{Q}$, and so we have found a path from $(\Phi, \Psi)$ to $(\Lambda, \Delta)$, proving that the latter is a flag of $\mathcal{P} \mid \mathcal{Q}$. The second part follows immediately from the first.

For the third part, we need to show that, given flags flags $(\Phi, \Psi)$ and $(\Lambda, \Delta)$ of $\mathcal{P} \mid \mathcal{Q}$, there is a path from $(\Phi, \Psi)$ to $(\Lambda, \Delta)$ that can be written as the concatenation of a path that never uses color $n$ with a path that never uses color $n-2$. The path described in the previous paragraph already satisfies this condition.

Theorem 4.5. Let $\mathcal{P}$ be a facet-bipartite $n$-polytope and let $\mathcal{Q}$ be a vertex-bipartite $m$ polytope. Let $\mathcal{M}=\mathcal{P} \mid \mathcal{Q}$. Then $\mathcal{M}$ is an $(m+n-1)$-polytope that is $(n-2, n)$-flat.

Proof. It is straightforward to check that if $i$ and $j$ are in $\{0, \ldots, m+n-2\}$ with $|i-j|>1$, then $\mathcal{M}[i, j]$ consists of 4 -cycles; this shows that $\mathcal{M}$ is a maniplex. Flatness was proved in Proposition 4.4. To show that $\mathcal{M}$ is a polytope, it suffices to show that it satisfies the Path Intersection Property. Consider two arbitrary flags of $\mathcal{M}$, say $(\Phi, \Psi)$ and $(\Lambda, \Delta)$. Suppose that there is a path from $(\Phi, \Psi)$ to $(\Lambda, \Delta)$ that uses only colors in $\{0, \ldots, j\}$ and another 
path that uses only colors in $\{i, \ldots, m+n-2\}$. We want to show that there must be a path that uses only the colors $\{i, \ldots, j\}$.

Since colors greater than $n-1$ do not affect the first component, the path that uses colors in $\{i, \ldots, m+n-2\}$ induces a path in $\mathcal{P}$ from $\Phi$ to $\Lambda$ that uses colors in $\{i, \ldots, n-1\}$. Since colors less than $n-1$ do not affect the second component, following the same sequence of colors in $\mathcal{M}$ gives us a path from $(\Phi, \Psi)$ to either $(\Lambda, \Psi)$ or $\left(\Lambda, \Psi^{0}\right)$. In the latter case, we can follow one more edge of color $n-1$ to arrive at $\left(\Lambda^{n-1}, \Psi\right)$. Now, the path from $(\Phi, \Psi)$ to $(\Lambda, \Delta)$ that uses colors in $\{0, \ldots, j\}$ induces a path from $\Psi$ to $\Delta$ that uses colors in $\{n-1, \ldots, j\}$, and following this sequence of colors in $\mathcal{M}$ gives us a path from wherever we stopped (either $(\Lambda, \Psi)$ or $\left.\left(\Lambda^{n-1}, \Psi\right)\right)$ to either $(\Lambda, \Delta)$ or $\left(\Lambda^{n-1}, \Delta\right)$. Since we supposed that $(\Lambda, \Delta)$ was a flag of $\mathcal{M}$, Proposition 4.4 implies that $\left(\Lambda^{n-1}, \Delta\right)$ is not a flag of $\mathcal{M}$, and so we must have arrived at $(\Lambda, \Delta)$. Thus, we have a path from $(\Phi, \Psi)$ to $(\Lambda, \Delta)$ that only uses colors in $\{i, \ldots, n-1\} \cup\{n-1, \ldots, j\}=\{i, \ldots, j\}$, as desired.

Example 4.6. If $\mathcal{Q}$ is a $k$-gon with $k$ even, then $\mathcal{P}|\mathcal{Q} \cong \mathcal{P}| k$. Essentially, each flag of the $k$-gon corresponds to a choice of one of the $k$ layers and one of the colors red or blue.

Proposition 4.7. Let $\mathcal{P}$ be a facet-bipartite $n$-polytope and let $\mathcal{Q}$ be a vertex-bipartite $m$ polytope. If $\mathcal{Q}$ is facet-bipartite, then $\mathcal{P} \mid \mathcal{Q}$ is facet-bipartite.

Proof. If there is a cycle in $\mathcal{P} \mid \mathcal{Q}$ with an odd number of edges labeled $m+n-2$, this induces a cycle in $\mathcal{Q}$ with an odd number of edges labeled $m-1$.

Proposition 4.7 implies that, if $\mathcal{Q}_{1}, \ldots, \mathcal{Q}_{k}$ are all vertex-bipartite and facet-bipartite, then we may construct a flat amalgamation $\mathcal{P}\left|\mathcal{Q}_{1}\right| \cdots \mid \mathcal{Q}_{k}$.

Finally, let us determine the automorphism group of $\mathcal{P} \mid \mathcal{Q}$. Given an automorphism $\varphi$ of $\mathcal{P}$ that sends $\Phi$ to $\Lambda$, let us say that $\varphi$ is $(n-1)$-even (respectively $(n-1)$-odd) if the number of edges labeled $n-1$ in any path from $\Phi$ to $\Lambda$ is even (respectively odd). (As long as $\mathcal{P}$ is facet-bipartite, this is well-defined.) We will similarly define automorphisms of $\mathcal{Q}$ to be 0 -even or 0 -odd.

Theorem 4.8. Let $\mathcal{P}$ be a facet-bipartite $n$-polytope with base flag $\Phi$ and let $\mathcal{Q}$ be a vertexbipartite $m$-polytope with base flag $\Psi$. Then

$$
\Gamma(\mathcal{P} \mid \mathcal{Q})=\{(\varphi, \psi) \in \Gamma(\mathcal{P}) \times \Gamma(\mathcal{Q}): \varphi \text { is }(n-1) \text {-even if and only if } \psi \text { is } 0 \text {-even }\}
$$

In particular, if all automorphisms of $\mathcal{P}$ are $(n-1)$-even and all automorphisms of $\mathcal{Q}$ are 0 -even, then $\Gamma(\mathcal{P} \mid \mathcal{Q})=\Gamma(\mathcal{P}) \times \Gamma(\mathcal{Q})$, and otherwise $\Gamma(\mathcal{P} \mid \mathcal{Q})$ is an index-2 subgroup of $\Gamma(\mathcal{P}) \times \Gamma(\mathcal{Q})$.

Proof. Clearly, each automorphism of $\Gamma(\mathcal{P} \mid \mathcal{Q})$ induces an automorphism $\varphi$ of $\mathcal{P}$ and an automorphism $\psi$ of $\mathcal{Q}$, and so $\Gamma(\mathcal{P} \mid \mathcal{Q}) \leq \Gamma(\mathcal{P}) \times \Gamma(\mathcal{Q})$. Conversely, given automorphisms $\varphi$ and $\psi$, we may try to build an automorphism $(\varphi, \psi)$ of $\mathcal{P} \mid \mathcal{Q}$ that acts component-wise. Clearly, this will only work if $(\Phi \varphi, \Psi \psi)$ is in $\mathcal{P} \mid \mathcal{Q}$, and this is true if and only if the parity 
of the number of edges labeled $n-1$ from $\Phi$ to $\Phi \varphi$ is the same as the parity of the number of edges labeled 0 from $\Psi$ to $\Psi \psi$. If that is the case, then note that for each flag $(\Lambda, \Delta)$,

$$
(\Lambda, \Delta)^{i}(\varphi, \psi)=\left(\Lambda^{i}, \Delta^{i-n+1}\right)(\varphi, \psi)=\left(\Lambda^{i} \varphi, \Delta^{i-n+1} \psi\right)=\left((\Lambda \varphi)^{i},(\Delta \psi)^{i-n+1}\right)=(\Lambda \varphi, \Delta \psi)^{i},
$$

proving that $(\varphi, \psi)$ is an automorphism. That proves the first part and the second follows immediately.

Example 4.9. Suppose $\mathcal{P}$ is the cuboctahedron and $\mathcal{Q}$ is its dual, the rhombic dodecahedron. Then $\mathcal{P}$ is facet-bipartite: we can color all of the square faces with one color and the triangles with another. Every automorphism of $\mathcal{P}$ is 2-even. Similarly, $\mathcal{Q}$ is vertex-bipartite, and its automorphisms are all 0-even. Thus $\Gamma(\mathcal{P} \mid \mathcal{Q})=\Gamma(\mathcal{P}) \times \Gamma(\mathcal{Q})$, a group of order $48^{2}$.

\section{Conclusions}

We have shown that every finite polytope $\mathcal{P}$ has a flat extension, where we glue together an even number of copies of $\mathcal{P}$ in a flat way. The strategy used does not work if we want to use an odd number of copies of $\mathcal{P}$. In particular, if we use an odd number of copies, then we cannot match each flag $\Phi_{i}$ to some $\Phi_{j}$ - some flags $\Phi_{i}$ must get matched to $\Psi_{j}$ with $\Phi \neq \Psi$. When is this possible and how can we do this in a consistent way?

Problem 1. Describe a construction that takes an $n$-polytope $\mathcal{P}$ and produces an $(n-2, n)$-flat $(n+1)$-polytope with 3 facets all isomorphic to $\mathcal{P}$. What restrictions on $\mathcal{P}$ are there?

Another interesting problem would be to further investigate the properties of the flat extensions that were described in Section 3.2.

Problem 2. Determine the automorphism group of the flat extensions described in Section 3.2.

\section{References}

[1] B. A. Anderson, Finite topologies and Hamiltonian paths, J. Combinatorial Theory Ser. B 14 (1973), 87-93. MR 312447

[2] Gabe Cunningham, Minimal equivelar polytopes, Ars Math. Contemp. 7 (2014), no. 2, 299-315. MR 3240439

[3] Gabe Cunningham, María Del Río-Francos, Isabel Hubard, and Micael Toledo, Symmetry type graphs of polytopes and maniplexes, Ann. Comb. 19 (2015), no. 2, 243-268. MR 3347382

[4] Gabe Cunningham and Daniel Pellicer, Open problems on k-orbit polytopes, Discrete Mathematics 341 (2018), no. 6, 1645 - 1661. 
[5] Jorge Garza-Vargas and Isabel Hubard, Polytopality of maniplexes, Discrete Mathematics 341 (2018), no. 7, $2068-2079$.

[6] A. Kotzig, Hamilton graphs and Hamilton circuits, Theory of Graphs and its Applications (Proc. Sympos. Smolenice, 1963), Publ. House Czechoslovak Acad. Sci., Prague, 1964, pp. 63-82. MR 0173249

[7] Peter McMullen and Egon Schulte, Abstract regular polytopes, Encyclopedia of Mathematics and its Applications, vol. 92, Cambridge University Press, Cambridge, 2002. MR 1965665 (2004a:52020)

[8] Mark Mixer, Egon Schulte, and Asia Ivić Weiss, Hereditary polytopes, Rigidity and symmetry, Fields Inst. Commun., vol. 70, Springer, New York, 2014.

[9] Alexander Rosa, Perfect 1-factorizations, Math. Slovaca 69 (2019), no. 3, 479-496. MR 3954017

[10] Steve Wilson, Maniplexes: Part 1: maps, polytopes, symmetry and operators, Symmetry 4 (2012), no. 2, 265-275. 